DOI: $10.21105 /$ joss. 03823

\title{
CCA-Zoo: A collection of Regularized, Deep Learning based, Kernel, and Probabilistic CCA methods in a scikit-learn style framework
}

\author{
James Chapman ${ }^{1}$ and Hao-Ting Wang ${ }^{2,3}$ \\ 1 Centre for Medical Image Computing, University College London, London, UK 2 Centre de \\ Recherche de I'Institut Universitaire de Gériatrie de Montréal, Université de Montréal, Montréal, \\ QC, Canada 3 Centre de Recherche de I'Hôpital du Sacré Coeur de Montréal, Université de \\ Montréal, Montréal, QC, Canada
}

\section{Software}

- Review ¿

- Repository ¿

- Archive ¿

Editor: Elizabeth DuPre ¿ Reviewers:

- Qrobbisg

- @hugorichard

- @ejolly

Submitted: 04 October 2021

Published: 18 December 2021

\section{License}

Authors of papers retain copyright and release the work under a Creative Commons Attribution 4.0 International License (CC BY 4.0).

\section{Summary}

Multi-view data has gained visibility in scientific research. Examples include different languages in natural language processing, as well as neuroimaging, multiomics and audiovisual data. Canonical Correlation Analysis (CCA) (Hotelling, 1992) and Partial Least Squares (PLS) are classical methods for investigating and quantifying multivariate relationships between these views of data. The goal of CCA and its variants is to find projections (and associated weights) for each view of the data into a latent space where they are highly correlated.

The original CCA is constrained by the sample-to-feature ratio. The algorithm cannot produce a solution when the number of features in one view exceeds the number of samples. To overcome this restriction, the original CCA has been developed into a family of models which include regularised (Vinod, 1976), kernelized (Hardoon et al., 2004), probabilistic/generative (Bach \& Jordan, 2005), and deep learning based (Andrew et al., 2013) variants. In particular these variations have allowed practitioners to apply these models to complex, high dimensional data. Similarly, variants of PLS have been proposed including the widely used Penalized Matrix Decomposition algorithm (Witten et al., 2009) which induces sparsity in the weight vectors for interpretability and generalisation.

cca-zoo is a Python package that implements many variants in a simple API with standardised outputs. We would like to highlight the unique benefits our package brings to the community in comparison to other established Python packages containing implementations of CCA. Firstly, cca-zoo contains a number of regularised CCA and PLS for high dimensional data that have previously only been available in installable packages in R. Native Python implementation will give Python users convenient access to these powerful models for both application and the development of new algorithms. Secondly,cca-zoo contains several deep CCA variants written in PyTorch (Paszke et al., 2019). We adopted a modular style allowing users to apply their desired neural network architectures for each view for their own training pipeline. Thirdly, cca-zoo contains generative models including probabilistic and deep variational CCA. This class of variations can be used to model the multiview data generation process and even generate new synthetic samples. Finally, cca-zoo provides data simulation utilities to synthesize data containing specified correlation structures as well as the paired MNIST data commonly used as a toy dataset in deep multiview learning.

Chapman et al., (2021). CCA-Zoo: A collection of Regularized, Deep Learning based, Kernel, and Probabilistic CCA methods in a scikit-learn 1 style framework. Journal of Open Source Software, 6(68), 3823. https://doi.org/10.21105/joss.03823 


\section{Statement of need}

The Python ecosystem for multiview learning currently provides a few options for implementing CCA and PLS models. scikit-learn (Pedregosa et al., 2011) contains standard implementations of both CCA and PLS for two-view data which plug into their mature API. pyrcca (Bilenko \& Gallant, 2016) contains implementations of ridge regularised and kernelized twoview CCA. The embed module of mvlearn (Perry et al., 2020) is perhaps the closest relative of cca-zoo, containing implementations of ridge regularised and kernelized multi-view CCA. cca-zoo builds on the mvlearn API by providing an additional range of regularised models and in particular sparsity inducing models which have found success in multiomics. Building on the reference implementation in mvlearn, cca-zoo further provides a number of deep learning models with a modular design to enable users to supply their own choice of neural network architectures.

Standard implementations of state-of-the-art models help as benchmarks for methods development and easy application to new datasets. cca-zoo extends the existing ecosystem with a number of sparse regularised CCA models. These variations have found popularity in genetics and neuroimaging where signals are contained in a small subset of variables. With applications like these in mind, cca-zoo simplified the access to the learnt model weights to perform further analysis in the feature space. Furthermore, the modular implementations of deep CCA and its multiview variants allow the user to focus on architecture tuning. Finally, cca-zoo adds generative models including variational (C. Wang, 2007) and deep variational CCA (W. Wang et al., 2016) as well as higher order canonical correlation analysis with tensor (Kim et al., 2007) and deep tensor CCA (Wong et al., 2021).

\section{Implementation}

cca-zoo adopted a similar API to that used in scikit-learn. The user first instantiates a model object and its relevant hyperparameters. Next they call the model's fit() method to apply the data. After fitting, the model object contains its relevant parameters such as weights or dual coefficients (for kernel methods) which can be accessed for further analysis. For models that fit with iterative algorithms, the model may also contain information about the convergence of the objective function. After the model has been fit, its transform() method can project views into latent variables and score() can be used to measure the canonical correlations.

The deep and probabilistic models are supported by PyTorch and NumPyro respectively. Due to the size of these dependencies, these two classes of variations are not in the default installation. Instead, we provide options [deep] and [probabilistic] for users. The list bellow provides the complete collection of models along with their installation tag is provided below.

\section{Model List}

A complete model list at the time of publication:

\begin{tabular}{llll}
\hline Model Class & Model Name & Number of Views & Install \\
\hline CCA & Canonical Correlation & 2 & standard \\
rCCA & Analysis & & \\
KCCA & Canonical Ridge & 2 & standard \\
& Kernel Canonical & 2 & standard \\
MCCA & Correlation Analysis & & standard \\
& Multiset Canonical & $>=2$ &
\end{tabular}

Chapman et al., (2021). CCA-Zoo: A collection of Regularized, Deep Learning based, Kernel, and Probabilistic CCA methods in a scikit-learn 2 style framework. Journal of Open Source Software, 6(68), 3823. https://doi.org/10.21105/joss.03823 


\begin{tabular}{|c|c|c|c|}
\hline Model Class & Model Name & Number of Views & Install \\
\hline $\mathrm{KMCCA}$ & $\begin{array}{l}\text { Kernel Multiset Canonical } \\
\text { Correlation Analysis }\end{array}$ & $>=2$ & standard \\
\hline GCCA & $\begin{array}{l}\text { Generalized Canonical } \\
\text { Correlation Analysis }\end{array}$ & $>=2$ & standard \\
\hline KGCCA & $\begin{array}{l}\text { Kernel Generalized } \\
\text { Canonical Correlation } \\
\text { Analysis }\end{array}$ & $>=2$ & standard \\
\hline PLS & Partial Least Squares & $>=2$ & standard \\
\hline CCA_ALS & $\begin{array}{l}\text { Canonical Correlation } \\
\text { Analysis by Alternating } \\
\text { Least Squares) (Golub \& } \\
\text { Zha, 1995) }\end{array}$ & $>=2$ & standard \\
\hline PLS_ALS & $\begin{array}{l}\text { Partial Least Squares by } \\
\text { Alternating Least Squares) }\end{array}$ & $>=2$ & standard \\
\hline PMD & $\begin{array}{l}\text { Sparse CCA by Penalized } \\
\text { Matrix Decomposition }\end{array}$ & $>=2$ & standard \\
\hline ElasticCCA & $\begin{array}{l}\text { Sparse Penalized CCA } \\
\text { (Waaijenborg et al., 2008) }\end{array}$ & $>=2$ & standard \\
\hline ParkhomenkoCCA & $\begin{array}{l}\text { Sparse CCA } \\
\text { (Parkhomenko et al., } \\
2009 \text { ) }\end{array}$ & $>=2$ & standard \\
\hline SCCA & $\begin{array}{l}\text { Sparse Canonical } \\
\text { Correlation Analysis by } \\
\text { Iterative Least Squares } \\
\text { (Mai \& Zhang, 2019) }\end{array}$ & $>=2$ & standard \\
\hline SCCA_ADMM & $\begin{array}{l}\text { Sparse Canonical } \\
\text { Correlation Analysis by } \\
\text { Altnerating Direction } \\
\text { Method of Multipliers } \\
\text { (Suo et al., 2017) }\end{array}$ & $>=2$ & standard \\
\hline SpanCCA & $\begin{array}{l}\text { Sparse Diagonal Canonical } \\
\text { Correlation Analysis } \\
\text { (Asteris et al., 2016) }\end{array}$ & $>=2$ & standard \\
\hline SWCCA & $\begin{array}{l}\text { Sparse Weighted } \\
\text { Canonical Correlation } \\
\text { Analysis (Wenwen et al., } \\
\text { 2018) }\end{array}$ & $>=2$ & standard \\
\hline TCCA & $\begin{array}{l}\text { Tensor Canonical } \\
\text { Correlation Analysis }\end{array}$ & $>=2$ & standard \\
\hline KTCCA & $\begin{array}{l}\text { Kernel Tensor Canonical } \\
\text { Correlation Analysis (Kim } \\
\text { et al., 2007) }\end{array}$ & $>=2$ & standard \\
\hline DCCA & $\begin{array}{l}\text { Deep Canonical } \\
\text { Correlation Analysis }\end{array}$ & $>=2$ & deep \\
\hline DCCA_NOI & $\begin{array}{l}\text { Deep Canonical } \\
\text { Correlation Analysis by } \\
\text { Non-Linear Orthogonal } \\
\text { Iterations (W. Wang, } \\
\text { Arora, Livescu, \& Srebro, } \\
\text { 2015) }\end{array}$ & $>=2$ & deep \\
\hline
\end{tabular}




\begin{tabular}{|c|c|c|c|}
\hline Model Class & Model Name & Number of Views & Install \\
\hline DCCAE & $\begin{array}{l}\text { Deep Canonically } \\
\text { Correlated Autoencoders } \\
\text { (W. Wang, Arora, } \\
\text { Livescu, \& Bilmes, 2015) }\end{array}$ & $>=2$ & deep \\
\hline DTCCA & $\begin{array}{l}\text { Deep Tensor Canonical } \\
\text { Correlation Analysis }\end{array}$ & $>=2$ & deep \\
\hline SplitAE & $\begin{array}{l}\text { Split Autoencoders } \\
\text { (Ngiam et al., 2011) }\end{array}$ & 2 & deep \\
\hline DVCCA & $\begin{array}{l}\text { Deep Variational } \\
\text { Canonical Correlation } \\
\text { Analysis }\end{array}$ & $>=2$ & deep \\
\hline ProbabilisticCCA & $\begin{array}{l}\text { Probabilistic Canonical } \\
\text { Correlation Analysis }\end{array}$ & 2 & probabilistic \\
\hline
\end{tabular}

\section{Documentation}

The package is accompanied by documentation (https://cca-zoo.readthedocs.io/en/latest/ index.html) and a number of tutorial notebooks which serve as both guides to the package as well as educational resources for CCA and PLS methods.

\section{Conclusion}

cca-zoo fills many of the gaps in the multiview learning ecosystem in Python, including a flexible API for deep-learning based models, regularised models for high dimensional data (and in particular those that induce sparsity), and generative models.cca-zoo will therefore help researchers to apply and develop Canonical Correlation Analysis and Partial Least Squares models. We continue to welcome contributions from the community.

\section{Acknowledgements}

JC is supported by the EPSRC-funded UCL Centre for Doctoral Training in Intelligent, Integrated Imaging in Healthcare ( i4health) (EP/S021930/1) and the Department of Health's NIHR-funded Biomedical Research Centre at University College London Hospitals. HTW is supported by funds from la Fondation Courtois awarded to Dr. Pierre Bellec.

\section{References}

Andrew, G., Arora, R., Bilmes, J., \& Livescu, K. (2013). Deep canonical correlation analysis. International Conference on Machine Learning, 1247-1255.

Asteris, M., Kyrillidis, A., Koyejo, O., \& Poldrack, R. (2016). A simple and provable algorithm for sparse diagonal CCA. International Conference on Machine Learning, 1148-1157.

Asteris, M., Kyrillidis, A., Koyejo, O., \& Poldrack, R. (2016). A simple and provable algorithm for sparse diagonal CCA. International Conference on Machine Learning, 1148-1157.

Bach, F. R., \& Jordan, M. I. (2005). A probabilistic interpretation of canonical correlation analysis. https://statistics.berkeley.edu/sites/default/files/tech-reports/688.pdf 
Bilenko, N. Y., \& Gallant, J. L. (2016). Pyrcca: Regularized kernel canonical correlation analysis in python and its applications to neuroimaging. Frontiers in Neuroinformatics, 10, 49. https://doi.org/10.3389/fninf.2016.00049

Golub, G. H., \& Zha, H. (1995). The canonical correlations of matrix pairs and their numerical computation. In Linear algebra for signal processing (pp. 27-49). Springer. https: //doi.org/10.1007/978-1-4612-4228-4_3

Hardoon, D. R., Szedmak, S., \& Shawe-Taylor, J. (2004). Canonical correlation analysis: An overview with application to learning methods. Neural Computation, 16(12), 2639-2664. https://doi.org/10.1162/0899766042321814

Hotelling, H. (1992). Relations between two sets of variates. In Breakthroughs in statistics (pp. 162-190). Springer. https://doi.org/10.2307/2333955

Kim, T.-K., Wong, S.-F., \& Cipolla, R. (2007). Tensor canonical correlation analysis for action classification. 2007 IEEE Conference on Computer Vision and Pattern Recognition, 1-8. https://doi.org/10.1109/cvpr.2007.383137

Mai, Q., \& Zhang, X. (2019). An iterative penalized least squares approach to sparse canonical correlation analysis. Biometrics, 75(3), 734-744. https://doi.org/10.1111/biom.13043

Ngiam, J., Khosla, A., Kim, M., Nam, J., Lee, H., \& Ng, A. Y. (2011). Multimodal deep learning. ICML.

Parkhomenko, E., Tritchler, D., \& Beyene, J. (2009). Sparse canonical correlation analysis with application to genomic data integration. Statistical Applications in Genetics and Molecular Biology, 8(1). https://doi.org/10.2202/1544-6115.1406

Paszke, A., Gross, S., Massa, F., Lerer, A., Bradbury, J., Chanan, G., Killeen, T., Lin, Z., Gimelshein, N., Antiga, L., \& others. (2019). Pytorch: An imperative style, highperformance deep learning library. Advances in Neural Information Processing Systems, 32, 8026-8037.

Pedregosa, F., Varoquaux, G., Gramfort, A., Michel, V., Thirion, B., Grisel, O., Blondel, M., Prettenhofer, P., Weiss, R., Dubourg, V., \& others. (2011). Scikit-learn: Machine learning in python. The Journal of Machine Learning Research, 12, 2825-2830.

Perry, R., Mischler, G., Guo, R., Lee, T., Chang, A., Koul, A., Franz, C., Richard, H., Carmichael, I., Ablin, P., \& others. (2020). Mvlearn: Multiview machine learning in python. arXiv Preprint arXiv:2005.11890.

Suo, X., Minden, V., Nelson, B., Tibshirani, R., \& Saunders, M. (2017). Sparse canonical correlation analysis. arXiv Preprint arXiv:1705.10865.

Vinod, H. D. (1976). Canonical ridge and econometrics of joint production. Journal of Econometrics, 4(2), 147-166. https://doi.org/10.1016/0304-4076(76)90010-5

Waaijenborg, S., Witt Hamer, P. C. V. de, \& Zwinderman, A. H. (2008). Quantifying the association between gene expressions and DNA-markers by penalized canonical correlation analysis. Statistical Applications in Genetics and Molecular Biology, 7(1). https://doi. org/10.2202/1544-6115.1329

Wang, C. (2007). Variational bayesian approach to canonical correlation analysis. IEEE Transactions on Neural Networks, 18(3), 905-910. https://doi.org/10.1109/tnn.2007. 891186

Wang, W., Arora, R., Livescu, K., \& Bilmes, J. (2015). On deep multi-view representation learning. International Conference on Machine Learning, 1083-1092.

Wang, W., Arora, R., Livescu, K., \& Srebro, N. (2015). Stochastic optimization for deep CCA via nonlinear orthogonal iterations. 2015 53rd Annual Allerton Conference on Communication, Control, and Computing (allerton), 688-695. https://doi.org/10.1109/allerton. 2015.7447071 
Wang, W., Yan, X., Lee, H., \& Livescu, K. (2016). Deep variational canonical correlation analysis. arXiv Preprint arXiv:1610.03454.

Wenwen, M., Juan, L., \& Zhang, S. (2018). Sparse weighted canonical correlation analysis. Chinese Journal of Electronics, 27(3), 459-466. https://doi.org/10.1049/cje.2017.08.004

Witten, D. M., Tibshirani, R., \& Hastie, T. (2009). A penalized matrix decomposition, with applications to sparse principal components and canonical correlation analysis. Biostatistics, 10(3), 515-534. https://doi.org/10.1093/biostatistics/kxp008

Wong, H. S., Wang, L., Chan, R., \& Zeng, T. (2021). Deep tensor CCA for multi-view learning. IEEE Transactions on Big Data. 